\title{
Effect of Docetaxel Combined with Cisplatin Preoperative Neoadjuvant Chemotherapy for Stage III NSCLC
}

\author{
Guocheng Ma1, Wei Chen¹ and Minquan Ma² \\ Department of Respiratory $1 /$ Emergency², Lanzhou General Hospital, Lanzhou Military Area Command,730000,China
}

\begin{abstract}
The aim of this study was to investigate the effect of docetaxel plus cisplatin preoperative neoadjuvant chemotherapy in stage III non-small cell lung cancer (NSCLC). It was an analytical study carried out from May 2013 to May 2017. Eightytwo patients with stage III NSCLC were divided into Group A and B according to a random number table $(n=41)$. Group A received neoadjuvant chemotherapy with docetaxel plus cisplatin before surgery. Group B underwent surgical treatment after preoperative examination. Compared with group B,operation time of group A was shorter $(p<0.001)$; intraoperative blood loss of group A was lower $(p<0.001)$; surgical resection rate and 1-year survival rate in group A were higher $(p=0.013,0.023)$. This suggested that preoperative neoadjuvant chemotherapy with docetaxel plus cisplatin may improve surgical resection rate, shorten operation time, reduce intraoperative blood loss, and prolong survival of patients.
\end{abstract}

Key Words: Docetaxel, Cisplatin, Neoadjuvant chemotherapy, Stage III non-small cell lung cancer.

How to cite this article: Ma G, Chen W, Ma M. Effect of docetaxel combined with cisplatin preoperative neoadjuvant chemotherapy for stage III NSCLC. J Coll Physicians Surg Pak 2019; 29(12):1230-1231.

Lung cancer is a very common malignant tumor of the respiratory system. Non-small cell lung cancer (NSCLC) accounts for $80 \%$ of lung cancers, while $30 \%$ of patients with NSCLC are at a late stage (stage III) at onset. ${ }^{1}$ Patients with stage III NSCLC are prone to recurrence or metastasis after surgery. Docetaxel is a semi-synthetic derivative of paclitaxel, and its anti-tumor effect is related to the microtubule network, interfering with the mitotic function of cells. ${ }^{2}$ Cisplatin is a platinum complex that is a non-specific drug in the cell cycle. Cisplatin is able to inhibit growth and reproduction of tumor cells. ${ }^{3}$ The combination of docetaxel and cisplatin has a significant synergistic effect. Chemotherapy has a therapeutic effect on local disease and micrometastases, but only leads to the death of chemotherapy-sensitive cells. Residual insensitive or resistant cells only be removed by surgery. Related data show that preoperative neoadjuvant chemotherapy may treat existing micrometastasis as early as possible and reduce the incidence of distant metastasis after surgery. ${ }^{4}$ Aim of this study was to investigate effect of docetaxel plus cisplatin preoperative neoadjuvant chemotherapy in stage III NSCLC.

This study was carried out at the Respiratory Department, Lanzhou General Hospital, Lanzhou Military Area Command, China, from May 2013 to May 2017. Eighty-

Correspondence to: Minquan Ma, Department of Emergency,

Lanzhou General Hospital of Lanzhou Military Region,

730000, China

E-mail:tvm611@163.com

Received: December 31, 2018; Revised: May 15, 2019;

Accepted: June 26, 2019 two patients with stage III NSCLC were selected. Inclusion criteria were confirmed stage IIla NSCLC; patients below the age of 70; no distant metastasis; and the karnofsky performance status score 70 or more. Exclusion criteria were patients with severe heart, liver, and kidney disease, and those who had undergone chemoradiotherapy. The patients were divided into Group $A$ and Group B with 41 cases in each group according to the method of random number table. Group A received neoadjuvant chemotherapy with docetaxel plus cisplatin before surgery. The protocol used was that intravenous infusion of $75 \mathrm{mg} / \mathrm{m}^{2}$ docetaxel on the first day, intravenous infusion of $25 \mathrm{mg} / \mathrm{m}^{2}$ cisplatin on the $2^{\text {nd }}-5^{\text {th }}$ day, 3 weeks for a course of treatment, a total of 2 courses. Auxiliary treatments such as routine hydration, liver protection, and stomach protection were performed. Three weeks after the end of chemotherapy, all patients in the study group underwent surgery 3 weeks after the end of chemotherapy. Group B underwent surgical treatment after preoperative examination. The same surgical treatment was used in both groups, i.e., patients underwent double-lumen endotracheal intubation, lobectomy, laparoscopic lobectomy, and pneumonectomy through the infraorbital/anterior lateral/posterior incision. All patients underwent lymph node dissection. Surgical resection rate, operative time and intraoperative blood loss were compared between the two groups. After 1 year of follow-up, the 1-year survival rate of the two groups was observed.

Data processing was performed using SPSS version 25 . Measurement data, like operation time and intraoperative blood loss, were expressed as mean \pm standard deviation and compared by independent 
Table I: Comparison of operative indexes between two groups.

\begin{tabular}{l|c|c|c}
\hline Parameter & Group A $(n=41)$ & Group B $(n=41)$ & $p$-value \\
\hline Operation time $(\min )$ & $109.37 \pm 17.41$ & $148.16 \pm 23.25$ & $<0.001$ \\
Intraoperative blood loss $(\mathrm{ml})$ & $498.22 \pm 31.59$ & $602.85 \pm 51.64$ & $<0.001$ \\
Surgical resection rate $[\mathrm{n}(\%)]$ & $40(97.56)$ & $33(80.49)$ & 0.013 \\
1-year survival rate $[\mathrm{n}(\%)]$ & $39(95.12)$ & $32(78.05)$ & 0.023 \\
\hline
\end{tabular}

sample $t$ test. Count data was expressed in $n(\%)$ and Chi-square test was used. A value of $p<0.05$ was considered to be statistically significant.

Among eighty-two patients, $47(57.32 \%)$ were males and $35(42.68 \%)$ were females; with age of $42-69$ (57.04 $\pm 2.63)$ years; with weight of $45-68(61.57 \pm 1.86) \mathrm{kg}$; with 52 cases $(63.41 \%)$ of squamous cell carcinoma, 24 cases $(29.27 \%)$ of adenocarcinoma, 6 cases $(7.32 \%)$ of adenosquamous carcinoma.

Compared with group B,operation time of group A was shorter $(p<0.001)$; intraoperative blood loss of group $A$ was lower $(p<0.001)$; surgical resection rate and 1-year survival rate in group $A$ were higher $(p=0.013, p=0.023$ respectively, Table I). No treatment-related death occurred in both the groups. Allergic reaction and peripheral neurotoxicity were not found during the treatment. The main adverse reactions of the two groups were radiation esophagitis, radiation pneumonia and leukopenia, all of which were grade I-II adverse reactions, and no grade III-IV adverse reactions occurred.

A study showed that neoadjuvant docetaxel-cisplatin was effective and tolerable in potentially operable stage IIIA NSCLC. ${ }^{5}$ The results of this study indicated that docetaxel combined with cisplatin preoperative neoadjuvant chemotherapy improved surgical resection rate of patients, shortened operation time, reduced intraoperative blood loss, and improved 1-year survival rate. The conclusion of this study was basically consistent with Liao et al.6, who showed docetaxel combined with cisplatin preoperative neoadjuvant chemotherapy was both active and well tolerated in patients with stage IIIN2 NSCLC.

Possible causes include docetaxel combined with cisplatin preoperative neoadjuvant chemotherapy may effectively resist the differentiation, proliferation and infiltration of tumor cells; reduce local tumors, metastatic lymph nodes, tumor volume, the probability of tumor spread, staging of lung cancer in various parts of the body and clinical TNM staging, eliminate multidrug resistance of lung cancer and root cause of recurrence. Thus, patient's post-operative survival is improved.

In conclusion, presurgical treatment with docetaxel and cisplatin can play a synergistic role, and it is feasible to treat stage III NSCLC.

\section{CONFLICT OF INTEREST:}

Authors declared no conflict of interest.

\section{AUTHORS' CONTRIBUTION:}

GM: Drafting the work, the conception or design of the work; or the acquisition, analysis, or interpretation of data for the work.

WC: Revising it critically for important intellectual content. MM: The conception or design of the work, final approval of the version to be published.

\section{REFERENCES}

1. Gaur P, Bhattacharya S, Kant $S$, Kushwaha RAS, Singh G, Pandey S. EGFR mutation detection and its association with clinicopathological characters of lung cancer patients. World $J$ Oncol 2018; 9:151-5.

2. Borghaei H, Paz-Ares L, Horn L, Spigel DR, Steins M, Ready NE, et al. Nivolumab versus docetaxel in advanced nonsquamous non-small-cell lung cancer. N Engl J Med 2015; 373:1627-39.

3. Taira T, Yoh K, Nagase S, Kubota K, Ohmatsu H, Niho S, et al. Long-term results of S-1 plus cisplatin with concurrent thoracic radiotherapy for locally advanced non-small-cell lung cancer. Cancer Chemother Pharmacol 2018; 81:565-72.

4. Chen H, Liang $\mathrm{C}$, Zhang L, Huang S, Wu X. Clinical efficacy of modified preoperative neoadjuvant chemotherapy in the treatment of locally advanced (stage IB2 to IIB) cervical cancer: Randomized study. Gynecol Oncol 2008; 110:308-15.

5. Betticher DC, Hsu Schmitz SF, Tötsch M, Hansen E, Joss C, Von BC, et al. Mediastinal lymph node clearance after docetaxel-cisplatin neoadjuvant chemotherapy is prognostic of survival in patients with stage IIIA pN2 non-small-cell lung cancer: A multicenter phase II trial. J Clin Oncol 2003; 21: 1752-9.

6. Liao WY, Chen JH, Wu M, Shih JY, Chen KY, Ho CC, et al. Neoadjuvant chemotherapy with docetaxel-cisplatin in patients with stage III N2 non-small-cell lung cancer. Clin Lung Cancer 2013; 14:418-24. 